\section{ONOMAREDIN}

Revista semestral de lingüística, filología y traducción
PONTIFICIA UNIVERSIDAD CATÓLICA DE CHILE CATOLICA DE CHILE
FACULTAD DE LETRAS

\title{
Relación entre pensamiento crítico y complejidad discursiva en estudiantes universitarios*
}

Relationship between critical thinking and discourse complexity among university students

\section{Lilian Hammer Krawczyk \\ Universidad de La Serena \\ Chile}

\section{Cristián Noemi Padilla}

Universidad de La Serena

Chile

\section{(c) $\underset{\mathrm{BY}}{(\mathrm{i})} \bigodot_{\mathrm{ND}}$}

Lilian Hammer Krawczyk: Maestría en Estudios Latinoamericanos, Dirección de Postgrado, Universidad de La Serena, Chile. I Correo electrónico: Iilian.hammer@gmail.com

Cristián Noemi Padilla: Departamento de Artes y Letras, Facultad de Humanidades, Universidad de La Serena,

Chile. | Correo electrónico: cnoemi@userena.cl 


\section{Resumen}

El trabajo ha pretendido develar aspectos de la relación entre capacidad o nivel de pensamiento crítico y grado o complejidad discursiva formal del discurso de estudiantes universitarios. En general, dicha relación ha sido investigada muy superficialmente, considerando solo mínimas variables intervinientes y focalizando la atención preferentemente sobre la estructura sintáctica. Los datos se obtuvieron de dos fuentes: a) de la aplicación del test Critical Thinking Test (CTC) y b) de una tarea de escritura de un ensayo narrativo/argumentativo. Los resultados han permitido identificar una relación de asociación entre las variables. Igualmente han permitido conformar una teoría fundamentada inicial de las nociones de pensamiento crítico y complejidad discursiva.

Palabras clave: pensamiento crítico; complejidad discursiva; discurso; texto.

\section{Abstract}

This study is intended to reveal aspects of the relationship between critical thinking skills —or critical thinking level - and the complexity in university students' discourse. Traditionally, this relationship has not been studied in depth. Previous studies have only considered minimal variables, and have focused mainly on syntactic structure. Data for our study was obtained from two sources: a) results from the Critical Thinking Test (CTC) and b) a writing assignment, namely a narrative/argumentative essay. Our findings have enabled us to identify a direct association between "critical thinking" and "discourse complexity". Based on these findings, we have formed a preliminary theory on the nature of this relationship.

Keywords: critical thinking; discourse complexity; discourse; text.

\footnotetext{
* Este trabajo se enmarca en el proyecto de investigación FONDECYT regular N 1130584.
} 


\section{Introducción}

El trabajo ha procurado especificar aspectos de la relación existente entre capacidad o nivel de pensamiento crítico y nivel o grado de complejidad discursiva en estudiantes universitarios pertenecientes a la Universidad Santo Tomás, Universidad Central y Universidad de La Serena, de la IV región del país.

En términos generales, esta relación ha sido solo superficialmente explorada, considerando solo un conjunto muy limitado de variables intervinientes, y poniendo mayor énfasis sobre la estructura de superficie textual, particularmente respecto de la organización sintáctica (Véliz, 1999; Van Eemeren, Grootendorst y Snoeck, 2006).

A fin de alcanzar los propósitos, se analizó un corpus que emanó del resultado de un test aplicado a ochenta estudiantes y cuyo propósito fue medir tanto pensamiento crítico como complejidad discursiva, a fin de poder establecer habilidades cognitivas y lingüísticas diversas, expresadas en la predilección de la formación disciplinar de los estudiantes evaluados.

Develar una eventual relación entre pensamiento crítico y complejidad discursiva permitiría avanzar tanto en el conocimiento disponible respecto de los vínculos existentes entre la capacidad de pensamiento y de lenguaje, particularmente en lo que concierne a los módulos cognitivos específicos comprometidos en la relación (Fodor, 1986), como, a su vez, disponer de información relevante con vistas a establecer estrategias metodológicas para desarrollar la competencia cognitiva o lingüística.

En este tenor, el trabajo describe tanto aspectos del modo en que los estudiantes organizan su pensamiento en términos de lo que aquí consideramos capacidad o nivel de pensamiento crítico como características de la estructura formal del discurso, en términos de lo que se propone considerar el grado de complejidad discursiva.

\section{Marco teórico}

\subsection{Pensamiento crítico}

Los humanos somos seres de estructura muy compleja, simultáneamente determinados en nuestra conducta por instintos de diversa naturaleza (biopsicosociales) que dan lugar permanentemente a necesidades surgidas en contextos específicos y pautadas por un conjunto de reglas establecidas para su acceso, por lo que la satisfacción y gratificación de estas depende de nuestra capacidad para organizar adecuadamente nuestro comportamiento.

Esta organización requiere de un conjunto de habilidades y funciones cognitivas que evolucionan y se perfeccionan durante el desarrollo para acceder progresivamente, desde la dependencia más absoluta al momento de nacer, hacia un nivel de autonomía que permite la plena independencia de cada individuo en la interpretación, integración y valoración de sus experiencias y de sus respuestas frente a las contingencias del entorno.

Durante los primeros años de vida, los seres humanos asumimos que la representación mental de la realidad corresponde exactamente a la realidad misma (etapa de realismo absoluto) y, además, creemos que la verdad existe y es objetiva y absoluta, por lo que no hay reflexión alguna acerca de la construcción de conocimientos.

Con el desarrollo, tanto de las funciones cognitivas como de los rasgos de personalidad (de acuerdo tanto con las normas universales de la evolución humana como de las características particulares y maduración de cada sujeto), aparece, entre las funciones cognitivas, la de metacognición y, con ella, cierta capacidad reflexiva (pensamiento crítico) que permite generar y cuestionar argumentos, primero ajenos y en ciertas áreas de contenido, para luego transferir esa competencia tanto a diversos contextos y a las propias creencias como al propio proceso de construcción de conocimiento. 
La noción de pensamiento crítico ha sido abordada básicamente desde tres perspectivas diferenciadas. En primer lugar, encontramos un enfoque filosófico que enfatiza la calidad del pensamiento desde un punto de vista normativo, en términos de estándares o reglas, i. e., exactitud, claridad, imparcialidad, precisión, lógica, amplitud, relevancia, etc., y que está centrado en el pensador para describir sus características y habilidades, concebidas como cualidades, i. e., autocontrol emocional, agudeza perceptiva, coraje intelectual, mente abierta, habilidad inquisitiva, etc.

Trabajos como los de Paul y Elder (2005), Bailin (2002), Lipman (1991), Ennis (2013), Siegel (2013), entre otros, son representativos de este enfoque epistémico. La crítica más común que recibe este acercamiento es que desarrolla su investigación sobre un ideal, lo perfecto, lo que debería ser y no sobre el comportamiento real del ser humano en una situación concreta y un contexto sociocultural determinado.

Un segundo enfoque (Halpern, 2006; Sternberg, 2012), derivado de los postulados de la psicología cognitiva, por otra parte, ha centrado su atención en el sujeto real, a través de la investigación del tipo de procesamiento de información propio del pensador crítico según el tipo de conductas que ejecuta durante la actividad crítica, para poder distinguir y describir sus fases, tales como análisis, interpretación, definición del problema, formulación de hipótesis, etc.

La objeción central que se le ha formulado a este enfoque es que lo que propone, en rigor, es definir un patrón de ciertos pasos que, habitualmente, sigue un pensador crítico. En este sentido podría decirse que no necesariamente cualquier persona que siga todos los pasos para pensar críticamente obtendría como producto un pensamiento crítico realmente, pues el que la información sea procesada en ciertas etapas no es necesariamente lo que más incide en que el producto mental tenga determinadas características.
El tercer enfoque de aproximación ha sido el que surge desde la pedagogía, a partir del cual se clasifican las habilidades cognitivas de manera jerárquica en taxonomías, abarcando las primeras desde las consideradas como habilidades básicas, por ejemplo, comprender, hasta las últimas, consideradas habilidades cognitivas de orden superior, por ejemplo, analizar.

En este marco, en general, se asume que las tres destrezas cognitivas superiores que constituyen el pensamiento crítico son análisis, síntesis y evaluación o valoración (Bloom, 1956). La crítica principal a este enfoque es que se focaliza solo en las habilidades (aproximación muy limitada) y, sobre todo, que homologa algunas habilidades con pensamiento crítico, suponiendo que todo aquel que haya desarrollado y practique esas tres habilidades sería, entonces, un pensador crítico, lo que no ocurre necesariamente de modo empírico.

Las principales diferencias entre los enfoques se dan en torno a cuatro problemáticas centrales. La primera de ellas se refiere al rol de la disposición como elemento constitutivo del pensamiento crítico; la segunda, a si es una habilidad general o se desarrolla asociada a dominios específicos; en tercer lugar, la discusión gira en torno a si es una competencia transferible a diferentes contextos; y por último, respecto de la relevancia del rol de los parámetros o estándares de calidad.

Las diferentes propuestas tienen en común, no obstante, que en todas ellas se consideran elementos constitutivos del pensamiento crítico tres características básicas: una actividad cognitiva de tipo reflexivo, una determinada disposición o actitud y, finalmente, cierto conocimiento del contexto.

Según sabemos, el pensamiento reflexivo, al ser metacognitivo, solo puede darse cuando el contenido mental está registrado simbólicamente, lo que comienza a ocurrir recién a partir de los 18/24 meses de edad, cuando el desarrollo del lenguaje permite la representación lingüísti- 
ca y la construcción de conceptos, facultando la gradual abstracción de la experiencia, actividad cognitiva realizada a partir de lo simbólico.

Cuando se ponen en relación estos conceptos entre sí para obtener una determinada conclusión construimos una suerte de argumentos (que pueden o no formalizarse verbalmente) y que son expresión del contenido mental mismo. Sostenemos que es, precisamente, a partir de estos contenidos con los que se teje la cosmovisión individual y cultural en la interacción social que realiza cada sujeto.

En el marco de nuestro trabajo, admitimos que el pensamiento crítico es, en consecuencia, la acción de enjuiciar deliberadamente el conocimiento disponible, el que, al registrarse simbólicamente en un código lingüístico, se expresa en la forma de discurso, por lo que el análisis de con- tenido permite evaluar, al menos parcialmente, la calidad de aquel en cuanto competencia.

\subsection{Complejidad discursiva}

Por medio del concepto de complejidad discursiva hemos pretendido referir el resultado del conjunto de conocimientos que guardan relación con la capacidad estratégica de que disponen los hablantes para organizar, en distintos grados de textura o densidad, un discurso en lo que concierne al nivel macroestructural del mismo. De acuerdo con Van Dijk (1992), la macroestructura es una representación abstracta, en un sentido bottom up del contenido semántico del discurso. En términos cognitivos, representa una operación de reducción de la información de la estructura de superficie discursiva, como se muestra en la tabla 1, a partir de la novela "El ingenioso hidalgo Don Quijote de La Mancha".

\section{TABLA 1}

Representación de macroestructura

El ingenioso hidalgo Don Quijote de la Mancha

ME: Hidalgo se vuelve loco

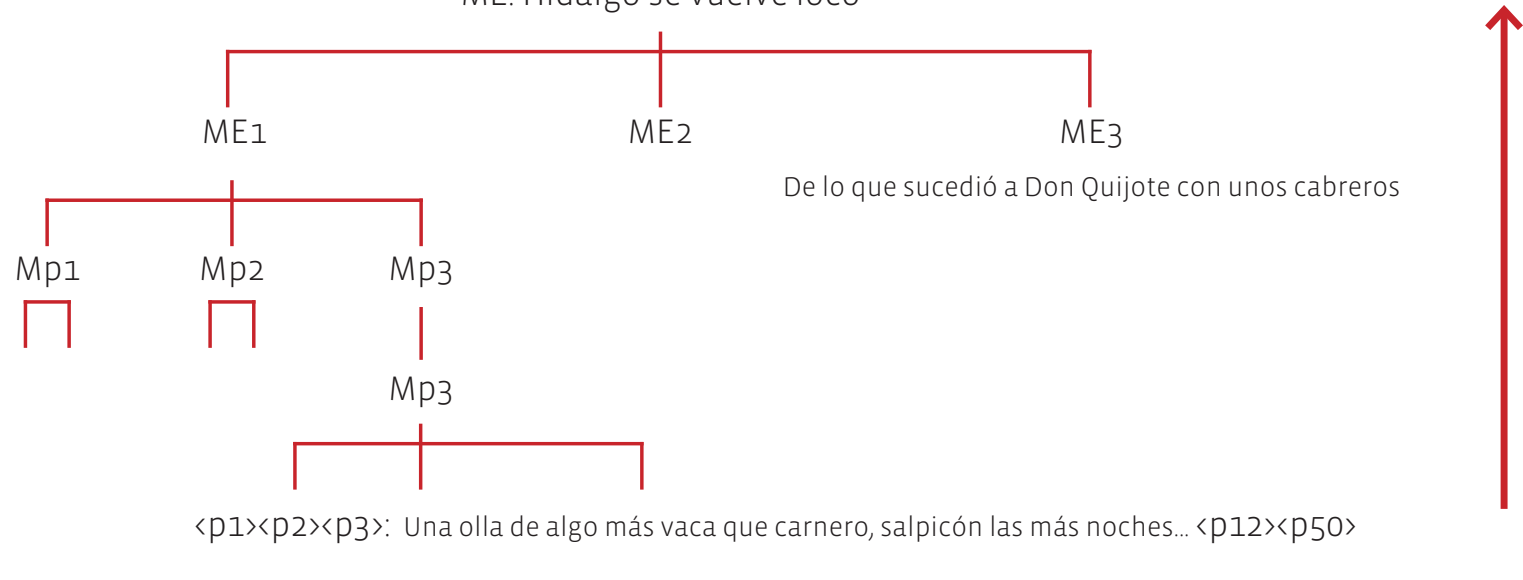

La manera en que se estructura el conjunto de proposiciones que conforma la macroestructura general del discurso parece indicar que la complejidad, textura o densidad discursiva depende de la puesta en ejecución del conocimiento concreto que suponen los saberes lingüísticos expresivo y elocutivo (Coseriu, 1992).
El conjunto de conocimientos que suponen la competencia expresiva y la competencia elocutiva, no obstante, resulta ser de naturaleza muy variada y de alcances muy diversos sobre la estructura lingüística, en términos de su textura, densidad o complejidad. Concebida la capacidad discursiva como una competencia, se impone para los propósitos de este trabajo, no obstante, 
esclarecer el tipo de conocimiento involucrado en estos saberes (i. e., pensamiento crítico) que se emplea para desplegar discursivamente la macroestructura, en distintos niveles de complejidad, en un sentido direccional top down, por medio de un conjunto de proposiciones sucesivamente de menor rango jerárquico.

Admitimos que las operaciones de textualización que suponen la conformación de la densidad, textura o complejidad discursiva tienen un sentido precisamente inverso al de las macrorreglas que proponen Van Dijk y Kintsch (1983), cuyo propósito, según su parecer, refieren el principio general que ha de seguirse para proyectar (levantar) la macroestructura de un discurso.

Este tipo de reglas, para nosotros una suerte de macrorreglas de densidad, textura o complejidad discursiva, suponen, en términos amplios, que la información general que comprende la macroestructura se especifica discursivamente en niveles macroestructurales cada vez menores hasta llegar al nivel microestructural donde ya no resulta posible más particularización temática.

Las reglas de producción discursiva que contribuyen a la densidad, textura o complejidad discursiva que admitimos y que constituyen, por su parte, una operación en sentido opuesto al de las macrorreglas, son básicamente: adjuntar, particularizar y especificar (Noemi, en prensa).

La regla adjuntar, como se grafica en la tabla 2, representa la operación contraria a la macrorregla omitir. En conformidad con la regla, una macroproposición, 〈Mpa〉, se despliega discursivamente en la macroestructura en una serie de proposiciones menores $\langle\mathrm{PC}\rangle,\langle\mathrm{Pd}\rangle,\langle\mathrm{Pn}\rangle$. A través de su aplicación, podemos arropar el discurso con una serie de proposiciones que no son relevantes en relación con el levantamiento de la macroestructura y que, según nuestra opinión, precisamente por ello pueden interpretarse como un índice menor de densidad, textura o complejidad discursiva, el que, además, debiera observarse en un escaso nivel de profundidad macroestructural.

\section{TABLA 2}

Macrorregla adjuntar

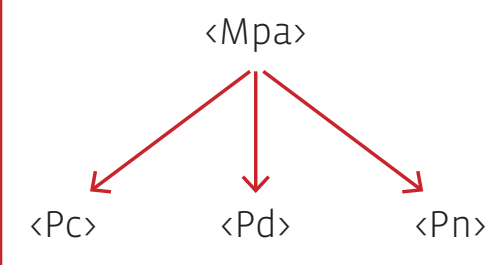

La regla particularizar, como pretende referir la tabla 3, representa la operación contraria a la regla generalizar. De este modo, a través de su aplicación, podemos descomponer una macroproposición $\langle\mathrm{Mpb}$ > en una serie de proposiciones menores $\left\langle P b_{1}\right\rangle$, $\left\langle P b_{2}\right\rangle$, $\left\langle P b_{3}\right\rangle$ que, a diferencia de la regla adjuntar, la implican. En cuanto regla de textualización, la regla especificar aparentemente resulta ser de mayor capacidad de densidad, textura o complejidad que la regla adjuntar.

\section{TABLA 3}

Macrorregla particularizar

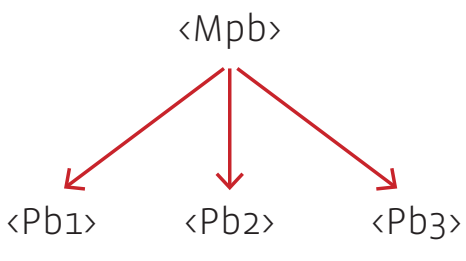

La regla especificar, finalmente, la concebimos como la regla inversa de la operación de integrar. Mediante su ejecución, una macroproposición correspondiente a un marco cultural $<M p$ marco a> se despliega discursivamente en una serie de proposiciones subsumidas en el mismo marco $\langle\mathrm{Pa}$ marco a $\rangle,\langle\mathrm{Pb}$ marco $\mathrm{a}\rangle,\langle\mathrm{Pn}$ marco a . Mediante la puesta en ejecución de esta regla, como se pretende representar en la tabla 4, podemos generar discurso en términos de proposiciones que estén subsumidas bajo el marco cultural o guión correspondiente en que se inscribe la macroestructura. Por su naturaleza, tanto particularizar como especificar son operaciones que debieran expresarse discursivamente en niveles macroestructurales de mayor profundidad. 


\section{TABLA 4}

Macrorregla especificar

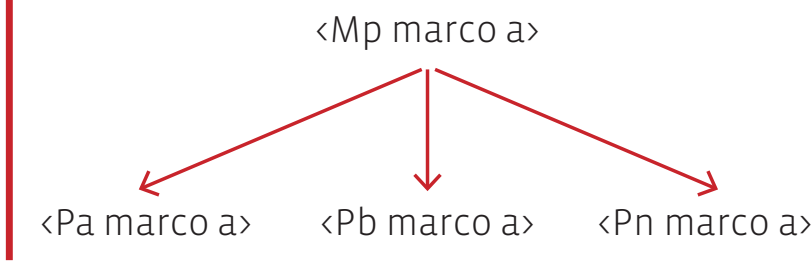

Tal cual se ha aceptado respecto del sentido en que operan las macrorreglas de levantamiento de información, debemos señalar, a su vez, que las reglas de generación discursiva no se consideran excluyentes entre sí, sino que se conciben en términos de una actuación complementaria y recursiva.

De esta forma, estimamos que a partir de la aplicación de las macrorreglas de densidad, textura o complejidad discursiva satisfacemos parte de las constricciones impuestas sobre los actos de habla en general, obteniendo, en consecuencia, un conjunto de proposiciones dispuestas en la forma de un discurso que alcanza un determinado grado de adecuación, en dependencia de su mayor o menor complejidad.

\subsubsection{Modelo normativo}

Como se ha esbozado en 2.2, concebimos que la competencia lingüística (Coseriu, 1992) se encuentra conformada por la suma de una serie de saberes independientes que han de interconectarse necesariamente entre sí en el momento en que aquella se actualiza en la forma de un discurso concreto.

Constreñida por la fuerza de una determinada tradición escritural, la competencia se proyecta desigualmente en la forma de discurso, afectando por ello la densidad, textura o complejidad macroestructural del mismo y, consecuentemente, su grado de coherencia.

Empleamos aquí el término coherencia para hacer mención a una interrelacionalidad global (macroestructural) en el texto (Halliday y Hasan,
1976) que es dependiente de la estructura formal y de las relaciones que establecen las partes del mismo. En este sentido, cabe señalar que estas relaciones no son lineales y se establecen en distintos niveles de profundidad del discurso (Van Dijk y Kintsch, 1983).

El discurso constituye, en este sentido, un juego de relaciones en el cual las partes actúan como marcadores o indicadores que pautan la densidad, textura o complejidad de la macroestructura: "El 'cosmos' del texto está constituido por esta 'textura' o juego de enlaces semántico-pragmáticos, dispuestos en diversas capas que se integran unas en otras" (Calsamiglia y Tusón, 2002: 219). Dependiendo del tipo de texto, diferentes marcadores o indicadores contribuirán a la mayor o menor coherencia del discurso. En el caso de nuestra investigación, el tipo textual ensayo faculta a que el generador de discurso conforme un texto con alta densidad o textura y, en consecuencia, contribuya a la coherencia global del mismo.

En dependencia del grado de variables del pensamiento crítico que posea o active un determinado hablante se actualizará, en conformidad, un tipo de discurso con mayor o menor grado de coherencia global determinada por la estructura textual del mismo y las relaciones de sus partes, potencialmente medible en términos de complejidad o intensidad de la textura del entramado discursivo.

Hemos supuesto, en consecuencia, variables normativas para el establecimiento de la complejidad discursiva del texto, tales como la longitud de las cadenas, las potenciales relaciones establecidas entre ellas, los niveles de profundidad de las mismas, etc. Para los efectos de este trabajo, tal cual se pretende representar en la tabla 5, sugerimos como marcadores de la complejidad discursiva (Noemi, 2013, 2014): el número de proposiciones $(P)$ por secuencia discursiva, el número desupport por secuencia discursiva, el número de explain por secuencia discursiva, el número de justify por secuencia discursiva y el nivel de profundidad macroestructural global. 
(P), proposición, representa el contenido semántico de una oración y, por tanto, constituye una unidad del nivel inferior de la macroestructura, asociada con la regla de textualización adjuntar y con la dimensión comunicación del test de pensamiento crítico.

Support representa un apoyo argumental de la (P); supone por parte del hablante mayor compromiso cognitivo y está probablemente asociado a mayor nivel de pensamiento crítico.

Explain constituye una explicación extraída a partir del estado de mundo discursivo expuesto por la (P) y avalada por support; supone igualmente actividad cognitiva de mayor envergadu- ra que, junto con support, parecen asociadas con la dimensión cognitiva de indagación medida por el test de pensamiento crítico.

Justify, finalmente, representa una justificación extraída a partir del mundo discursivo generado por $(P)$, support y explain. Supone el mayor esfuerzo cognitivo y se asocia con la dimensión de indagación que mide el test de pensamiento crítico.

Las macrorreglas de textualización particularizar y especificar, debido a su condición de recursividad, parecen asociarse a los niveles de profundidad macroestructural representados por, support, explain y justify.

\section{TABLA 5}

Representación de niveles de complejidad discursiva

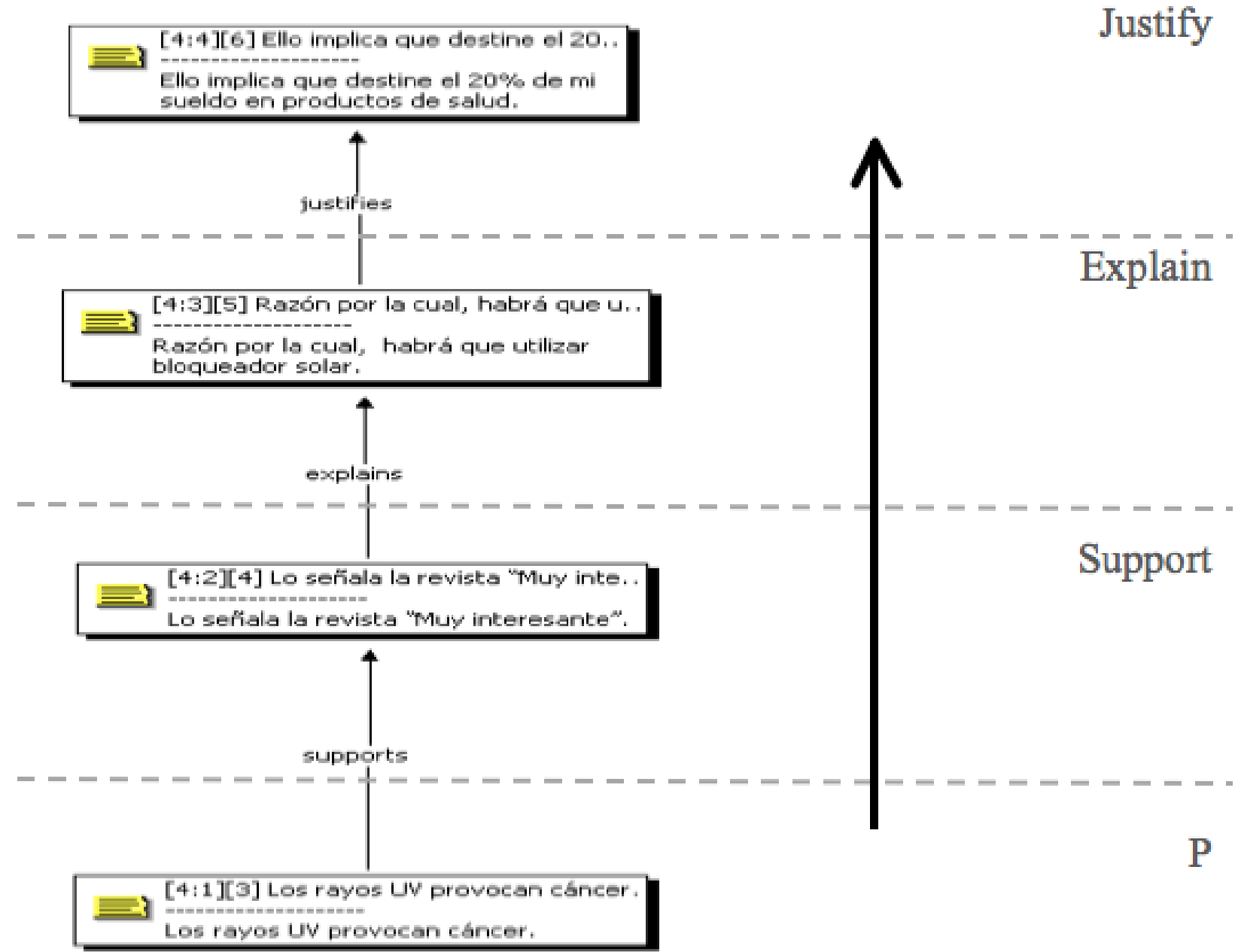




\section{Metodología}

En esta investigación, de carácter exploratorio, hemos seguido un enfoque de naturaleza mixta, cuantitativo y cualitativo.

\subsection{Instrumentos}

A fin de establecer la capacidad o nivel de pensamiento crítico de los estudiantes evaluados se empleó como instrumento de medición la prueba conocida como Tasks in Critical Thinking (TCT), creada en 1986 por una comisión experta del Educational Testing Service, a solicitud del The College Board de Estados Unidos y validada para Chile (Jelvez y Zambrano, 2005).

El test TCT fue aplicado a un total de 82 estudiantes, de entre 18 a 23 años, de las carreras de Pedagogía en Castellano y Filosofía, Ingeniería Comercial, Psicología y Sociología, pertenecientes a la Universidad Santo Tomás, Universidad Central y Universidad de La Serena, de la IV región del país.

El instrumento considera tanto la multidimensionalidad del pensamiento crítico como las destrezas cognitivas a priori que este requiere, en una prueba con quince reactivos de tipo analítico organizados según cinco subescalas y documentados a partir de tres textos en que el sujeto evalúa a partir de una tarea dada. EI TCT permite identificar las capacidades en aptitudes de razonamiento superior, utilizadas para analizar y resolver problemas, a través de tres dimensiones: comunicación, análisis e indagación.

Los quince reactivos, a través de los cuales se puede alcanzar un puntaje máximo de 35 puntos, están organizados en tres subescalas: (a) Indagación, a través de la cual se pretende establecer cuán diestro es un individuo para planear y organizar una búsqueda, comprender, extraer ideas, clasificar y evaluar información; (b) Análisis, cuyo propósito es discriminar cuán capaz es un sujeto para formular hipótesis y estrategias de análisis, aplicar técnicas, reglas y modelos para resolver problemas, demostrar flexibilidad y creatividad, conjeturar, evidenciar y encontrar relaciones, para así, finalmente sacar conclusiones; y (c) Comunicación, cuyo fin es establecer cuán hábil es un individuo para organizar una presentación, escribir efectivamente y comunicar información cuantitativa o visual.

Con el propósito de determinar el grado de complejidad discursiva de los informantes, hemos seguido un procedimiento cualitativo, aplicándose los procedimientos de la grounded theory (Glaser y Strauss, 1967), a través del software Atlas/ti. Con vistas a obtener un texto con predominancia de secuenciaciones argumentativas (Adam, 1992) por parte de los estudiantes informantes, se les solicitó de manera sucesiva a la aplicación del TCT la redacción de un ensayo, a partir de información proporcionada en el mismo test.

De acuerdo con el modelo, los textos se sintetizaron en categorías conceptuales que aparecieron contextualmente y a las cuales se les asignó un código abierto (Strauss y Corbin, 1998; Rodríguez y García, 1996). Posteriormente, se procedió a agrupar los códigos según sus propiedades; de esta forma se pudo obtener un conjunto de códigos que conformó una unidad superior mediante el recurso de una codificación axial, obteniéndose como resultado una aproximación al grado de complejidad discursiva de los informantes.

De manera emergente, en consecuencia, se obtuvieron tanto las categorías de medición de la complejidad discursiva de los informantes, en términos de (P), support, explain y justify, como una representación de la complejidad discursiva específica de cada uno de ellos expresada en la suma de los tipos de relaciones que cada uno los sujetos produjo en sus escritos (número total de (P), número total de support, número total de explain y número total de justify).

\section{Resultados y discusión 4.1. Presentación de los resultados}

Para estos efectos, en primer lugar se expresó operativamente la noción de pensamiento crítico en términos del puntaje total de cada sujeto, 
y la complejidad discursiva como la suma total de los tipos de relaciones que los sujetos producen en sus discursos. En primer lugar, se exploraron los datos de forma cuantitativa, pudiendo comprobarse que estos no permitían observar correlación (r) ni determinación (R2) entre las dos variables. En igual sentido, tampoco pudo observarse dependencia sistemática entre ellas (Chi Cuadrado). Se procedió luego, de forma cualitativa, a ordenar y puntuar en cuartiles o medios los puntajes de cada uno de los instrumentos. La exploración de la relación entre el pensamiento crítico y complejidad discursiva se llevó a cabo ubicando a cada sujeto en una gradiente. En consecuencia, se obtuvieron frecuencias brutas de los resultados.

\subsection{Resultados generales}

Con el propósito de determinar alguna tendencia en la relación observada a partir del análisis de casos, se procedió a agrupar los resultados totales de la muestra, obtenidos mediante ambos instrumentos, en una escala de cuartiles, según se puede observar en la tabla 6 .

A partir de lo representado en la tabla 6, resulta posible comprobar que, en general, hay muy pocos sujetos de la muestra que manifiestan alta complejidad discursiva (16 casos), en tanto que la mayoría de los discursos generados por los estudiantes revela baja complejidad (66 casos). En igual sentido es dable observar que los discursos de los sujetos con mayor puntaje en el
TCT (12 casos) manifiestan baja complejidad discursiva.

Los datos muestran que los sujetos que producen discursos con alta o muy alta complejidad discursiva tienen asociado un alto o muy alto rendimiento en el TCT (el 87,5\% de los casos). En igual sentido, se observa que la mayoría de los sujetos de la muestra manifiesta un alto o muy alto rango de pensamiento crítico (78,04\%), mientras que solo una minoría detenta un grado alto o muy alto de complejidad discursiva (21, 95\%). La información permite, de otra parte, comprobar que la mayoría de los sujetos con un rendimiento alto o muy alto en el TCT (64 casos) producen escritos con baja o muy baja complejidad (50 casos).

\subsection{Análisis de casos}

Con el propósito de evidenciar la relación entre capacidad o nivel de pensamiento y grado o complejidad discursiva, se presentan a continuación dos ejemplos prototípicos del corpus con los puntajes obtenidos en las dimensiones pensamiento crítico y complejidad discursiva, respectivamente.

El sujeto 1 representado en la tabla 7 puntúa como deficiente en términos de la dimensión de pensamiento crítico al alcanzar un puntaje total de 17 puntos, 8 en la categoría de análisis, 5 en la de indagación y un comportamiento normal (4 puntos) en la de comunicación.

\section{TABLA 6}

Complejidad discursiva (resultados totales expresados en cuartiles)

\begin{tabular}{|c|c|c|c|c|c|}
\hline TCT & MUY ALTA & ALTA & BAJA & MUY BAJA & TOTAL GENERAL \\
\hline MUY ALTA & & & 3 & 9 & 12 \\
\hline ALTA & 4 & 10 & 14 & 24 & 52 \\
\hline BAJA & 1 & 1 & 4 & 8 & 14 \\
\hline MUY BAJA & & & 1 & 3 & 4 \\
\hline TOTAL GENERAL & 5 & 11 & 22 & 44 & 82 \\
\hline
\end{tabular}




\section{TABLA 7}

Dimensión pensamiento crítico, sujeto 1

\begin{tabular}{ll}
\hline Pensamiento crítico & Deficiente \\
\hline Puntaje total & 17 \\
\hline Categoría comunicación & Normal \\
\hline Categoría análisis & 8 Puntos \\
\hline Categoría indagación & 5 Puntos \\
\hline
\end{tabular}

El sujeto 1, a través de las operaciones cognitivas que hemos denominado macrorreglas de densidad, textura o complejidad discursiva, genera un discurso (cf. tabla 8) de tipo explicativo/argumentativo con 11 proposiciones $(P)$ en la base, apoyadas por 10 (P2) (rotuladas como support por el software) en el siguiente nivel de profundidad macroestructural. Es posible observar igualmente en tanto estrategia discursiva que algunas (P) están soportadas por más de una (P2). Con arreglo a la propuesta, el hablante manifiesta baja complejidad discursiva, y consecuentemente baja coherencia, en virtud del débil tejido, entramado o densidad discursiva, representado por la escasa profundidad macroestructural y la ausencia de relaciones sustantivas entre proposiciones.

\section{TABLA 9}

Dimensión pensamiento crítico, sujeto 2

\begin{tabular}{ll}
\hline Categoría pensamiento crítico & Alto \\
\hline Puntaje total & 31 \\
\hline Categoría comunicación & Alto \\
\hline Categoría análisis & 12 Puntos \\
\hline Categoría indagación & 14 Puntos \\
\hline
\end{tabular}

El sujeto 2, por el contrario (cf. tabla 9), alcanza un puntaje alto en términos de la dimensión de pensamiento crítico, con un total de 31 puntos: 12 en la categoría de análisis, 14 en la de indagación y un desempeño alto en la de comunicación (5 puntos).

Como podemos observar en la tabla 10, el sujeto 2, por medio de las macrorreglas de densidad, textura o complejidad discursiva, genera a su vez un discurso de tipo explicativo/argumentativo con 3 proposiciones (P) en la base, soportadas por 4 (P2) en el segundo nivel de complejidad (rotulado por el software como support). Al contrario del sujeto 1 , este compromete mayor densidad discursiva al avanzar con macrorreglas de mayor envergadura cognitiva hacia un segundo

\section{TABLA 8}

Complejidad discursiva, sujeto 1

Support

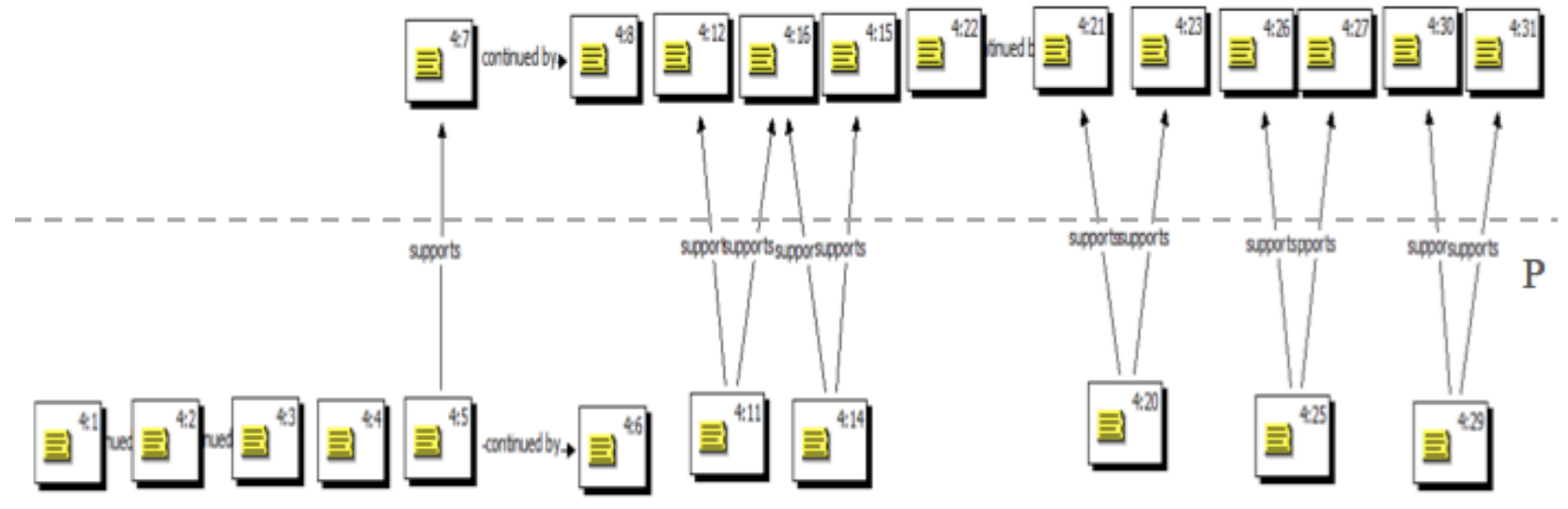


nivel de complejidad macroestructural, rotulado por el software como explain y que garantiza, en virtud de la textura, mayor coherencia global.

En efecto, las 4 (P2) están garantizadas por 7 (P3) que, a su vez, establecen diversas relaciones según se observa en la tabla 9 (cruces, haces, etc.). Es posible igualmente observar que el sujeto 2 aplica macrorreglas de aun mayor compromiso cognitivo hasta alcanzar un tercer nivel de densidad o complejidad discursiva rotulado como justifies por el software, tal cual es el caso de 2 ( $\left(P_{3}\right)$, por lo que, en términos de su textura, el discurso alcanza un grado de complejidad y coherencia estimables.

\section{Conclusiones}

El trabajo ha permitido poner de manifiesto inicialmente ciertas operaciones cognitivas asociadas con la competencia lingüística: adjuntar, particularizar y especificar, cuyo rol, como se ha sugerido, consiste en desplegar en el discurso en sentido top down la macroestructura textual, provocando, en consecuencia, distintos grados de densidad, textura o complejidad discursiva, con arreglo al nivel de pensamiento crítico que posea el sujeto enunciador y afectando por ello la coherencia.

El cruce de la información proporcionada por el test Task in Critical Thinking y la obtenida cualitativamente por medio del procedimiento establecido en la grounded theory nos ha permitido aproximarnos a aspectos de la relación entre pensamiento y lenguaje, particularmente en lo que guarda relación con los dominios específicos de los módulos de cognición involucrados.

A partir de la muestra sometida a análisis, es posible afirmar que existe relación de asociación entre capacidad o nivel de pensamiento crítico y complejidad discursiva. Esta corre desde la complejidad discursiva hacia el pensamiento crítico. Los sujetos de la muestra que manifiestan, en efecto, alta o muy alta complejidad discursiva presentan a su vez alto o muy alto pensamiento crítico, en términos generales.

\section{TABLA 10}

Complejidad discursiva, sujeto 2

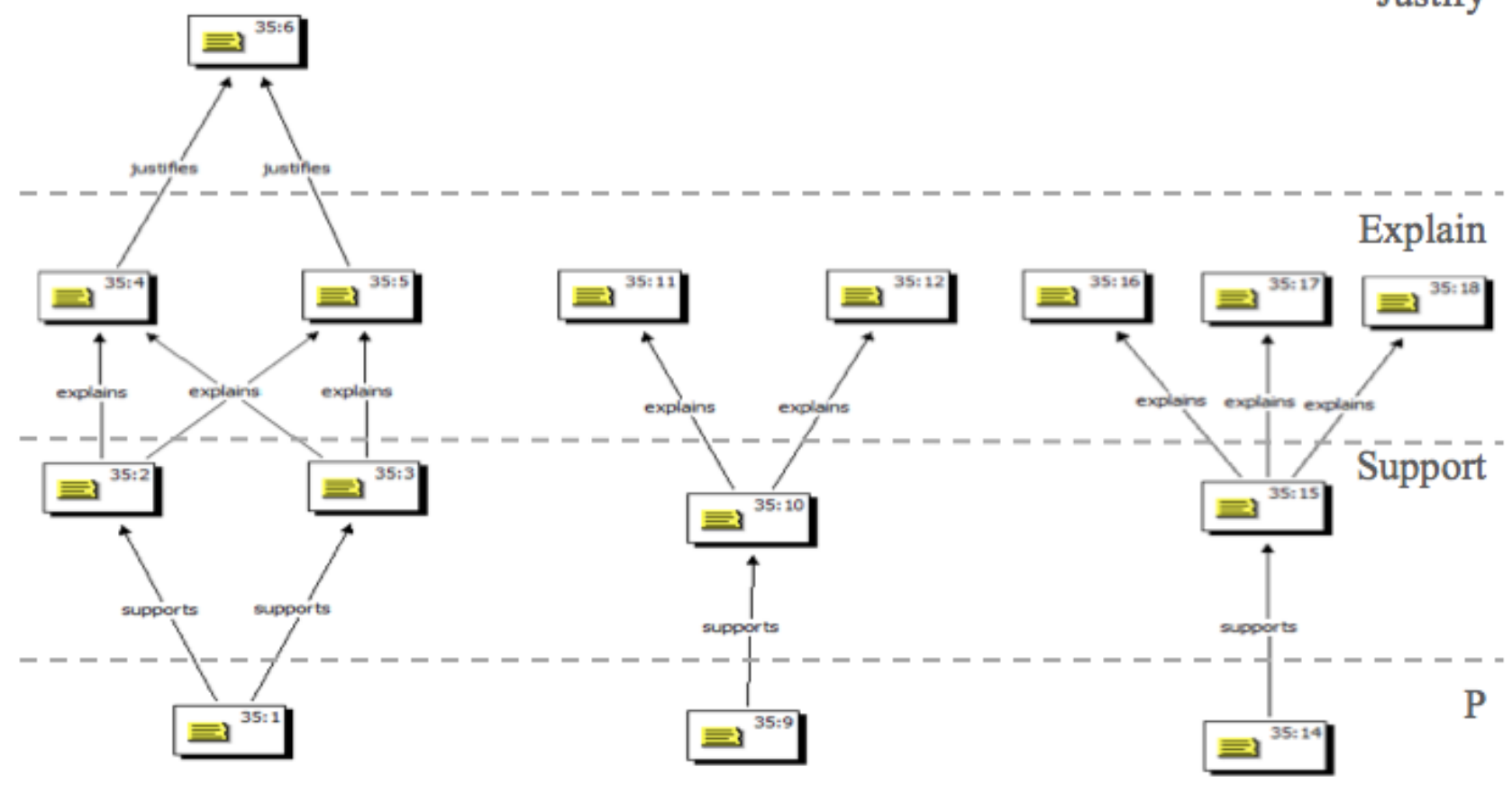

Justify 
La asociación inversa no es posible establecerla. Esto orienta la reflexión en el sentido de que aparentemente resulta más fácil adquirir pensamiento crítico que desplegarlo textualmente en la forma de un discurso. La operación de textualización, en otras palabras, compromete también otra u otras variables no descritas en este trabajo.

La condición de complejidad discursiva resulta ser la expresión de una competencia poco común que detentan ciertos sujetos que manifiestan características cognitivas representadas en un alto o muy alto grado de pensamiento crítico. Sin embargo, la condición de alto o muy alto grado de pensamiento crítico no es condición sine qua non de complejidad discursiva. La variable de complejidad discursiva, en este sentido, permite discriminar mucho más que la de pensamiento crítico.

Este trabajo constituye un aporte inicial en una línea de investigación que debiera, junto con incorporar corpus de distinta naturaleza, proporcionar en sucesivas aproximaciones evidencia respecto de la naturaleza de los nexos que vinculan las proposiciones durante el proceso de generación discursiva, los tipos de relaciones que se establecen entre aquellas y la justificación cognitiva que faculta determinado entramado discursivo.

\section{Bibliografía citada}

Adam, Jean-Michel, 1992: Les textes: tvpes et prototypes, Paris: Nathan.

Alexy, Robert, 1989: Teoría de la argumentación jurídica, Madrid: Centro de Estudios Constitucionales.

BAILIN, Sharon, 2002: "Critical thinking and science education", Science and Education, 11, 4, 361-375.

BLoom, Benjamin, y otros, 1956: Taxonomy of educational objectives: Handbook I. The cognitive domain, New York: David McKay \& Co.
Calsamiglia, Helena y Amparo Tusón, 2002: Las cosas del decir: manual de análisis del discurso, Barcelona: Ariel.

Coseriu, Eugenio, 1992: Competencia lingüística: elementos de la teoría del hablar, Madrid: Gredos.

EnNIs, Robert, 2013: "Critical thinking across the curriculum: The Wisdom CTAC Program", Inquiry: Critical Thinking across the Curriculum, 28, 2, 25-45.

Fodor, Jerry, 1986: La modularidad de la mente, Madrid: Ediciones Morata.

Glaser, Barney y Anselm Strauss, 1967: The discovery of grounded theory: strategies for qualitative research, New York: Aldine de Gruyter.

Halliday, Michael y Ruqaiya Hasan, 1976: Cohesion in English, London: Longman.

HALPERN, Diane, 2006: "The nature and nurture of critical thinking" en Robert SteRnberg, Henry RoeDIGER y Diane Halpern (eds.): Critical thinking in psychology, Cambridge, MA: Cambridge University Press, 1-14.

Jelvez, Marcela y Fabiola Zambrano, 2005: Pensamiento crítico en estudiantes de pedagogía en lenguaje y comunicación. Un estudio descriptivo. Tesis de licenciatura, Universidad Austral de chile.

LIPMAN, Matthew, 1991: Thinking in Education, New York: Cambridge University Press.

Noemi, Cristián, 2013: "Aproximación teórica a la noción de complejidad argumentativa", Logos: revista de lingüística, filosofía y literatura, 23, 2, 256-271.

Noemi, Cristián, 2014: "Un modelo de representación de complejidad argumentativa: el discurso de Friedman", Linguagem em (Dis)curso, 15, 2, 305-320.

Noemi, Cristián, en prensa: "Reasoning and argumentative complexity", Rozenberg Quarterly: the magazine, Amsterdam: Rozenberg Publisher. 
Paul, Richard y Linda Elder, 2005: The Thinker's Guide to the Nature and Functions of Critical and Creative Thinking, Dillon Beach, CA: Foundation for Critical Thinking.

Rodríguez, Gregorio, Javier Gil y Eduardo García, 1996: Metodología de la investigación cualitativa, Málaga: Aljibe.

Siegel, Harvey, 2013: "El pensamiento crítico como un ideal educacional", Logos: Revista de Lingüística, Filosofía y Literatura, 23, 2, 272-292.

SteRnBerg, Robert, 2012: "The assessment of creativity: An investment-based approach”, Creativity Research Journal 24, 3-12.

Strauss, Anselm y Juliet Corbin, 1998: Basics of qualitative research: techniques and procedures for developing grounded theory, London: Sage.

Van DıJk, Teun, 1992: La ciencia del texto, Barcelona: Ediciones Paidós.

Van DIJk, Teun y Walter KInTSCH, 1983: Strategies of discourse comprehension, New York: Academic Press.

Van Eemeren, Frans, Rob Grootendorst y Francisca Snoeck, 2006: Argumentación. Análisis, evaluación, presentación, Buenos Aires: Biblios.

Vélız, Mónica, 1999: "Complejidad sintáctica y modo del discurso", Estudios Filológicos 34,181192. 